J. Gastroenterol. Hepatol. Erkr. 2019 · 17:10-17 https://doi.org/10.1007/s41971-018-0043-9

Online publiziert: 8. Januar 2019

(c) Der/die Autor(en) 2019

CrossMark

Andreas Blesl ${ }^{1}$ Christoph Högenauer ${ }^{1}$ Robert Koch ${ }^{2}$ - Clemens Dejaco ${ }^{3}$. Harald Vogelsang ${ }^{3}$. Josef Stimakovits ${ }^{4} \cdot$ Harry Fuchssteiner $^{5}$. Thomas Haas ${ }^{6}$. Wolfgang Petritsch ${ }^{1}$

${ }^{1}$ Klinische Abteilung für Gastroenterologie und Hepatologie, Medizinische Universität Graz, Graz, Österreich

${ }^{2}$ Innere Medizin I, Medizinische Universität Innsbruck, Innsbruck, Österreich

${ }^{3}$ Klinische Abteilung für Gastroenterologie und Hepatologie, Innere Medizin III, Medizinische Universität Wien, Wien, Österreich

${ }^{4}$ Abteilung für Innere Medizin II, Gastroenterologie und Onkologie, Krankenhaus der Barmherzigen Brüder Eisenstadt, Eisenstadt, Österreich

${ }^{5}$ Innere Medizin 4, Gastroenterologie, Ordensklinikum Linz, Linz, Österreich

${ }^{6}$ Darm Praxis Salzburg, Salzburg, Österreich

\title{
Stellenwert von Vedolizumab als Erstlinienbiologikum bei chronisch-entzündlichen Darmerkrankungen (CED)
}

\section{Positionspapier der Arbeitsgruppe CED innerhalb der ÖGGH}

zumab als gleichwertige Option zu TNF$\alpha$-Inhibitoren ein $[2,3]$.

In einigen europäischen Ländern, wie z. B. Deutschland, der Schweiz und Kroatien, wurde die Erstattungsrichtlinie exakt der europäischen Zulassung angeglichen. In Österreich hingegen bedarf es für die Verordnung von Vedolizumab, wie in einigen anderen europäischen Ländern, wie z. B. in Spanien, Frankreich, Norwegen und Schweden, einer Vortherapie mit einem TNF- $\alpha$-Inhibitor oder des Bestehens von Kontraindikationen gegenüber diesen. In anderen Ländern, wie z. B. Italien, wurden bestimmte Kriterien festgelegt, bei denen eine Erstlinientherapie mit Vedolizumab erstattet wird.

Der Stellenwert von Vedolizumab zur Behandlung von CED wurde bereits ausführlich in einem Positionspapier der Arbeitsgruppe für CED innerhalb der Österreichischen Gesellschaft für Gastroenterologie und Hepatologie (ÖGGH) behandelt [4]. In der Zwischenzeit stehen weitere wertvolle Daten, insbesondere was den Einsatz als Erstlinientherapie und die Sicherheit betrifft, zur Verfü- gung, die ein Update des Positionspapiers notwendig erscheinen lassen.

Der aktuelle Stellenwert von Vedolizumab im Vergleich zu TNF- $\alpha$-Inhibitoren als Biologikaerstlinientherapie wird aus der Sicht der Arbeitsgruppe CED innerhalb der ÖGGH in Form von Statements festgehalten und entsprechend kommentiert.

\section{Statements}

1. Zur Induktionstherapie bei biologikanaiven Patienten mit moderater bis schwerer Colitis ulcerosa sind TNFa-Inhibitoren und Vedolizumab gleich wirksam. GEMINI I, die placebokontrollierte Zulassungsstudie für Vedolizumab bei Colitis ulcerosa, ist eine Induktionsund Erhaltungsstudie mit insgesamt 895 Patienten mit mittelschwerer bis schwerer aktiver Colitis ulcerosa nach Therapieversagen mit zumindest einer konventionellen Therapie (Glukokortikoide, Azathioprin, 6-Mercaptopurin) oder einem TNF- $\alpha$-Inhibitor. Die Wirksamkeit der Induktionstherapie wurde 
Tab. 1 Ansprechen und „mucosal healing“ bei TNF-naiven Colitis-ulcerosa-Patienten in den Zulassungsstudien zu Woche 6 bzw. Woche 8 [5-10]

\begin{tabular}{l|lll}
\hline Präparat & Studie & $\begin{array}{l}\text { Ansprechen } \\
\text { (Woche 6 bzw. 8; \%) }\end{array}$ & $\begin{array}{l}\text { "Mucosal healing" } \\
\text { (Woche 6 bzw. 8; \%) }\end{array}$ \\
\hline Infliximab & ACTI+ ACT II & 69 bzw. 65 & 62 bzw. 60 \\
\hline Adalimumab & ULTRA I+ II & 55 bzw. 59 & 47 bzw. 49 \\
\hline $\begin{array}{l}\text { Golimumab } \\
\text { Vedolizumab }\end{array}$ & PURSUIT & 51 & 42 \\
\hline & GEMINI I & 53 & 49
\end{tabular}

nach 6 Wochen bzw. 2 Gaben des Präparats evaluiert. Patienten, die Vedolizumab erhielten, hatten im Vergleich zu Placebo zur Woche 6 ein signifikant besseres klinisches Ansprechen (47\% vs. $26 \%$; $p<0,001)$ sowie eine höhere Rate an klinischer Remission (17\% vs. $5 \%$; $p=0,001)$ und „mucosal healing“ (41\% vs. $25 \%$; $p=0,001)$. Es ist darauf hinzuweisen, dass es sich bei den GEMINIDaten um ein teilweise (48\%) mit TNFa-Inhibitoren vorbehandeltes Kollektiv handelt [5].

Auch in den Zulassungsstudien für Infliximab bei Colitis ulcerosa, ACT I und ACT II, wurde die Überlegenheit von Infliximab gegenüber Placebo in Hinsicht auf die Ansprechraten zu Woche 8 demonstriert. Ein Ansprechen konnte in $69 \%$ bzw. $65 \%$ der Patienten, die mit einer Dosis von $5 \mathrm{mg} / \mathrm{kg}$ KG behandelt wurden, beobachtet werden. Der Unterschied zur Placebogruppe (37\% bzw. 29\% Ansprechen) war statistisch signifikant $(p<0,001)$. „Mucosal healing" $\mathrm{zu}$ Woche 8 wurde in $62 \%$ bzw. $60 \%$ der mit Infliximab behandelten Patienten beobachtet [6].

Für Adalimumab wurde in der ULTRA-II-Studie anhand von 518 Colitisulcerosa-Patienten die Überlegenheit des Präparats im Vergleich zu Placebo gezeigt. Ansprechen (50 \% vs. $35 \%$ ), Remission ( $17 \%$ vs. $9 \%)$ und „mucosal healing“ ( $41 \%$ vs. $32 \%)$ wurden signifikant öfter in der Adalimumabgruppe zu Woche 8 beobachtet $[7,8]$.

Die Zulassung für Golimumab für die Behandlung der Colitis ulcerosa erfolgte aufgrund der Daten der PURSUIT-Studie. Die Ansprechraten mit $51 \%$ zu Woche 6 in der Gruppe 200/100 mg waren im Vergleich zu $30 \%$ bei Placebo signifikant höher $(p<0,001)$. Auch klinische Remission und „mucosal healing“ wur- den in der Golimumabgruppe signifikant öfter beobachtet $(p<0,0014$; [9]).

Berücksichtigt man nur TNF-naive Patienten, zeigten sich für Infliximab Ansprechraten zu Woche 8 von $69 \%$ (ACT I) bzw. $65 \%$ (ACT II), für Adalimumab von $55 \%$ (ULTRA I) und $59 \%$ (ULTRA II) sowie für Golimumab (PURSUIT) zu Woche 6 von $51 \%$ und für Vedolizumab (GEMINI I) von 53\%. „Mucosal healing“ wurde mit Infliximab bei $62 \%$ bzw. $60 \%$, mit Adalimumab bei $47 \%$ bzw. $49 \%$, mit Golimumab bei $42 \%$ und mit Vedolizumab bei $49 \%$ der TNF-naiven Patienten erreicht (- Tab. 1; [5-10]).

Cholapranee et al. verglichen in einem rezent publizierten systematischen $\mathrm{Re}$ view die Studiendaten der TNF- $\alpha$-Inhibitoren und von Vedolizumab mittels einer Metaanalyse. Hierbei zeigt sich, dass Vedolizumab zur Induktion eines „mucosal healing" nach 6-12 Wochen genauso effektiv ist wie Golimumab (OR 1,15 [0,51-2,61]), Adalimumab (OR 1,42 $[0,70-2,94]$ ) und Infliximab (OR 0,63 [0,29-1,41]). Alle Therapien waren einem Placebo überlegen [11].

Selbiges Ergebnis brachte eine Metaanalyse von Danese et al. Auch hier zeigten sich keine weiteren signifikanten Unterschiede in der Wirksamkeit der TNFa-Inhibitoren im Vergleich zu Vedolizumab bezogen auf klinisches Ansprechen, die Remissionsrate und das „mucosal healing " bei Colitis-ulcerosa-Patienten [12].

Als Ausnahme zu oben genannten Daten muss hier die fulminante Colitis ulcerosa gesehen werden. Hierzu liegen keine Daten für Vedolizumab vor und die Gabe von intravenösen Kortikosteroiden sowie bei Versagen der Einsatz von Infliximab, Tacrolimus oder Cyclosporin sind laut ECCO-Leitlinie die State-ofthe-Art-Therapien für diese Indikation [2].
Als Limitation muss zu allen genannten Daten angemerkt werden, dass Headto-head-Studien mit direktem Vergleich der Biologika untereinander weiterhin fehlen und dass somit die Patientencharakteristika der verschiedenen, in den Reviews inkludierten Studien unterschiedlich sind und die Studien aus diesem Grund nicht uneingeschränkt vergleichbar sind.

2. In der Erhaltungstherapie bei Patienten mit Colitis ulcerosa mit initialem Response auf die verabreichte Biologikatherapie ist Vedolizumab den TNFa-Inhibitoren möglicherweise überlegen. In der GEMINI-I-Studie wurden Patienten, die auf die Vedolizumabtherapie zu Woche 6 angesprochen haben, in einem 1:1:1-Verhältnis neuerlich randomisiert. Die erste Gruppe erhielt nur Placebo, Gruppe 2 Vedolizumab alle 4 Wochen und Gruppe 3 Vedolizumab alle 8 Wochen. Patienten, die initial nicht auf die Vedolizumabtherapie angesprochen haben, erhielten Vedolizumab alle 4 Wochen als Erhaltungstherapie. Als primärer Endpunkt war die klinische Remission zu Woche 52 definiert. Der Endpunkt wurde in beiden Verumpatientengruppen signifikant öfters erreicht als in der Kontrollgruppe (Vedolizumab 8wöchentlich: 41,8\%; Vedolizumab 4-wöchentlich: 44,8\%; Placebo: 15,9\%; [5]). Im Vergleich hierzu liegen die Woche52- bzw. -54-Remissionsdaten für Infliximab (ACT I) bei $35 \%$ für die $5-\mathrm{mg} / \mathrm{kg}$ KG-Kohorte, für Adalimumab bei $17 \%$ (ULTRA II) und für Golimumab bei $34 \%$ (PURSUIT) [6, 13, 14]. Einschränkend muss zu diesen Daten allerdings erwähnt werden, dass in den Vedolizumab- und Golimumabzulassungsstudien nur die initialen Responder auf das Präparat (bei Vedolizumab $47 \%$, bei Golimumab $51 \%$, jeweils zu Woche 6) in die 52bzw. 54-Wochen-Analysen einflossen, während in ACT I und ULTRA II sämtliche initial eingeschlossene Patienten bis Woche 52 bzw. 54 verfolgt wurden. Des Weiteren waren in ULTRA II und PURSUIT Dosisadaptierungen erlaubt.

Vergleicht man die Langzeitdaten der am Markt befindlichen Biologika aus den Zulassungsstudien in Hinblick auf das „mucosal healing“ bei TNF-naiven Pati- 
enten nach 52 bzw. 54 Wochen, erkennt man einen Trend für einen Vorteil von Vedolizumab gegenüber den TNF- $\alpha$-Inhibitoren: Infliximab: $46 \%$ (ACT I; [6]), Adalimumab: $31 \%$ [13], Golimumab: $42 \%$ [14], Vedolizumab: $60 \%$ [10]. Beim Vergleich zum „mucosal healing“ gelten dieselben Einschränkungen bezüglich der Vergleichbarkeit der Studien wie für die Remissionsraten.

In einem rezenten systematischen Review verglichen Vickers et al. die Langzeitdaten der am Markt befindlichen Biologika zur CED-Therapie anhand von 5 Studien. Hierbei zeigte Vedolizumab in Bezug auf das klinische Langzeitansprechen nach 52 bzw. 54 Wochen signifikante Vorteile gegenüber den TNF- $\alpha$-Inhibitoren Infliximab (OR 3,18 [1,14-9,20]), Golimumab (OR 2,33 [1,04-5,41]) und Adalimumab (OR $3,96[1,67-9,84])$. Auch in Hinblick auf „mucosal healing“ war Vedolizumab Infliximab (OR 2,43 [0,87-6,66]) tendenziell und Adalimumab (OR 3,21 [1,33-7,35]) signifikant überlegen [15].

Diese Ergebnisse werden von Cholapranee et al. ebenfalls unterstützt. In Bezug auf den Erhalt des „mucosal healing" zeigte Vedolizumab tendenziell bessere Ergebnisse als Infliximab (OR 1,17 [0,35-3,84]), Adalimumab (OR 2,23 $[0,79-6,04])$ und Golimumab (OR 2,19 [0,69-6,96]; [11]).

Weiters zeigten auch Faleck et al. in einem bisher nicht veröffentlichen Abstract auf der ECCO 2018, dass bei 334 Colitisulcerosa-Patienten signifikant mehr Patienten nach 12 Monaten in der Vedolizumabgruppe eine klinische Remission (54\% vs. $37 \%$; HR $1,54[1,08-2,18])$ und ein „mucosal healing“ (50\% vs. $42 \%$, HR $1,73[1,10-2,73])$ hatten als Patienten unter TNF- $\alpha$-Inhibitor-Therapie [16]. Einschränkend muss man aber in Erinnerung rufen, dass es sich dabei um keine prospektive randomisierte Head-toHead-Studie handelt.

In einem Simulationsmodell mit 100.000 Patienten zur Identifizierung des idealen Einsatzzeitpunkts von Vedolizumab zur Therapie der Colitis ulcerosa konnte rezent gezeigt werden, dass der frühe Einsatzvon Vedolizumab vor anderen Therapieoptionen einen deutlichen Vorteil gegenüber dem Letztlinienein-
J. Gastroenterol. Hepatol. Erkr. 2019 · 17:10-17 https://doi.org/10.1007/s41971-018-0043-9 (c) Der/die Autor(en) 2019

A. BlesI · C. Högenauer · R. Koch · C. Dejaco · H. Vogelsang · J. Stimakovits · H. Fuchssteiner · T. Haas - W. Petritsch

Stellenwert von Vedolizumab als Erstlinienbiologikum bei
chronisch-entzündlichen Darmerkrankungen (CED).
Positionspapier der Arbeitsgruppe CED innerhalb der ÖGGH

Zusammenfassung

Vedolizumab (Entyvio ${ }^{\circledR}$ ) ist ein darmselektiver monoklonaler lgG1-Antikörper, der zur Behandlung der Colitis ulcerosa und des Morbus Crohn zugelassen ist. Im Vergleich zu Tumornekrosefaktor Alpha (TNF-a) Inhibitoren bietet Vedolizumab bei chronisch entzündlichen Darmerkrankungen (CED) vergleichbare Ansprechraten. Generell zeigt Vedolizumab für Patienten mit CED ein günstigeres Nebenwirkungsprofil hinsichtlich schwerer Nebenwirkungen und Infektionen, hat wahrscheinlich ein höheres Sicherheitsprofil im Alter und kann bei Kontraindikationen für TNF-a-Inhibitoren

wie z. B. höhergradige Herzinsuffizienz sicher eingesetzt werden. In Österreich ist derzeit die Erstattung limitiert auf Patienten mit Versagen, Unverträglichkeit oder Kontraindikationen auf eine Therapie mit TNF-aInhibitoren. Eine Erstlinienbiologikatherapie mit Vedolizumab bei Colitis ulcerosa oder Morbus Crohn ist laut Erstattungskodex derzeit nicht vorgesehen.

Schlüsselwörter

CED · Colitis ulcerosa - Morbus Crohn . Vedolizumab $\cdot$ Erstlinientherapie

\section{Status of Vedolizumab as First-Line Biologic Therapy for Inflammatory Bowel Disease (IBD). Position Paper from the Working Group IBD Within the ÖGGH}

\section{Abstract}

Vedolizumab (Entyvio ${ }^{\circledR}$ ) is a gut-selective monoclonal lgG1 antibody approved for the treatment of ulcerative colitis and Crohn's disease. Compared to TNF-a inhibitors, vedolizumab offers comparable response rates in patients with inflammatory bowel disease. In general, vedolizumab has a more favourable side effect profile for serious adverse events and infections, is likely to have a better safety profile in older age and can be safely used if contraindications for TNF-a inhibitors like a class III and IV congestive heart failure are present. In Austria, reimbursement is currently limited to TNF-a inhibitor failure, intolerance or patients with contraindications for these drugs. Vedolizumab as first-line biologic therapy for ulcerative colitis or Crohn's disease will currently not be reimbursed in Austria.

\section{Keywords}

IBD - Ulcerative colitis · Crohn's disease .

Vedolizumab · First-line therapy satz vor Kolektomie sowohl in Hinsicht auf die Remissionsrate als auch auf das Infektions- und Lymphomrisiko bringt. Je länger der Simulationszeitraum war, desto mehr kamen die Vorteile von Vedolizumab zum Tragen [17].

3. In der Erhaltungstherapie bei biologikanaiven Patienten mit Morbus Crohn bietet Vedolizumab im Allgemeinen vergleichbare Remissionsraten gegenüber anderen Biologikatherapien. Dies ist jedoch nicht für die Induktionstherapie zutreffend. In einer Posthoc-Analyse der Studien GEMINI II und III für Vedolizumab bei Morbus Crohn wurden insgesamt 1476 Patienten eingeschlossen. Es wurden sowohl TNFa-Therapieversager (960 Patienten) als auch TNF-naive Patienten (516 Patienten) inkludiert. Eine klinische Remission $\mathrm{zu}$ Woche 6 zeigten 22,7\% (TNF-naiv) bzw. $13,3 \%$ (TNF-vorbehandelt), zu Woche 52 waren es $48,9 \%$ bzw. $27,7 \%$ [18].

Danese et al. zeigten auf der ECCO 2018 in der VERSIFY-Studie, dass nach 26 Wochen ein Viertel der Patienten unter Vedolizumabtherapie ein endoskopisches Ansprechen zeigt, mit Vorteil für TNF-naive Patienten ( $28 \%$ vs. $22 \%$ bei mit TNF-vorbehandelten Patienten). $\mathrm{Zu}$ 
Hier steht eine Anzeige.

\section{曾 Springer}


einer vollständigen endoskopischen Heilung kommt es bei $24 \%$ (TNF-naiv) bzw. $7 \%$ (TNF-Versager) der Patienten [19].

In einem Review über 9 Studien mit insgesamt 2530 Patienten konnten Singh et al. betreffend die Induktionstherapie bei biologikanaiven Patienten mit Morbus Crohn zeigen, dass Vedolizumab Infliximab (OR 0,23 [0,06-0,78]) unterlegen und nicht signifikant unterschiedlich zu Adalimumab (OR 0,47 [0,13-1,75]) und Ustekinumab (OR 0,43 [0,09-2,23]) war. Nach einem Jahr Therapie mit Biologika bei Patienten mit initialem Ansprechen auf die jeweilige Therapie konnte bei Vedolizumab kein signifikanter Unterschied in der Wirksamkeit zu den anderen Präparaten festgestellt werden: Infliximab (OR 0,67 [0,06-5,64]), Adalimumab (OR 0,43 [0,05-3,36]), Ustekinumab (OR 0,87 [0,07-11,36]) [20].

Eine weitere Metaanalyse zu diesem Thema liegt von Hazlewood et al. vor. In dieser wurde Vedolizumab sowohl in der Induktion der Remission als auch in der Erhaltung dieser mit Infliximab, Adalimumab und Infliximab plus Azathioprin verglichen. Daten von Ustekinumab wurden zu diesem Zeitpunkt noch nicht berücksichtigt. Das Ergebnis zeigte, dass Vedolizumab in der Induktion zwar besser wirksam war als Placebo (OR 2,0 [1,2-3,3]), aber den anderen Präparaten tendenziell unterlegen war: Infliximab (OR 0,70 [0,25-1,5]), Adalimumab (OR $0,67[0,33-1,5])$, Infliximab plus Azathioprin (OR 0,47 $[0,18-1,1])$. In der Erhaltungstherapie zeigten sich für Vedolizumab Nachteile gegenüber Adalimumab (OR 0,42 [0,22-0,58]) und Infliximab plus Azathioprin (OR 0,42 [0,17-0,92]) und kein signifikanter Unterschied $\mathrm{zu}$ einer Infliximabmonotherapie (OR 0,77 [0,39-1,5]; [21]).

Ein etwas anderes Ergebnis zeigt ein ECCO-Abstract des heurigen Jahrs von Bohm et al. bei 538 nach Alter, Geschlecht, Hospitalisierungsrate, Strikturen, penetrierenden Komplikationen, Voroperationen, Krankheitsschweregrad, Steroidrefraktärität und TNFVersagen gemachten Morbus-CrohnPatienten. In dieser Kohorte hatten mit Vedolizumab behandelte Patienten signifikant höhere Raten an „mucosal healing" (50\% vs. $41 \%$; OR 1,67
$[1,13-2,47])$ und tendenziell bessere Raten an klinischer Remission (38\% vs. $34 \%$; OR 1,27 [0,91-1,78]) und steroidfreier Remission ( $26 \%$ vs. $18 \%$; OR 1,75 $[0,90-3,43])$ als Patienten unter Therapie mit TNF- $\alpha$-Inhibitoren. Auffällig war zusätzlich das signifikant bessere Ansprechen von Patienten mit Kolonbeteiligung auf Vedolizumab im Vergleich zu TNF- $\alpha$-Inhibitoren (klinische Remission: OR 1,51 [1,04-2,20]; steroidfreie Remission: OR 4,90 [2,44-9,83]; „,mucosal healing“: OR 1,70 [1,10-2,61]) [22]. Angemerkt muss hierbei werden, dass in der TNF- $\alpha$-Inhibitoren-Gruppe sowohl Patienten mit Adalimumab- als auch mit Infliximabtherapie inkludiert waren.

Weiters liegt ein rezenter systematischer Review zum Vergleich von Vedolizumab mit Ustekinumab bei Morbus Crohn und TNF- $\alpha$-Inhibitoren-Versagern vor, in denen Daten aus 5 Studien analysiert wurden. Sowohl in der Induktion der Remission (OR: 1,16 [0,54-2,48]) als auch im Erhalt dieser (OR: 0,72 [0,30-1,68]) waren beide Präparate nicht signifikant unterschiedlich wirksam [23].

Ein weiterer Aspekt, den Faleck et al. beleuchten konnten, ist, dass die Wirksamkeit von Vedolizumab bei Morbus Crohn umso höher ist, je kürzer die Erkrankung besteht. Wird das Präparat in den ersten beiden Erkrankungsjahren begonnen, befinden sich nach 6 Monaten $38 \%$ der Patienten in Remission. Bei späterem Beginn sind es nur noch $23 \%$ der Patienten [24].

\section{Vedolizumab ist bei Patienten mit Colitis ulcerosa und Morbus Crohn in Bezug auf schwere Nebenwirkungen und auf das Infektionsrisiko den TNF- a-Inhibitoren überlegen. Schon in der} Zulassungsstudie GEMINI I für Vedolizumab bei Colitis ulcerosa zeigte sich zwischen den verschiedenen Patientengruppen und $\mathrm{zu}$ Placebo kein signifikanter Unterschied an unerwünschten Nebenwirkungen. Auch schwere Nebenwirkungen und Infektionen waren in der Vedolizumabgruppe nicht häufiger und kein Fall von progressiver multifokaler Leukenzephalopathie (PML) trat auf. Bei der intravenösen Verabreichung des Präparats wurde keine Anaphylaxie dokumentiert [5].

In einem im Jahr 2018 publizierten systematischen Review verglichen Singh et al. unter anderem auch die Sicherheitsdaten der verschiedenen Biologika mittels Network-Metaanalyse bei Colitis ulcerosa. Für Vedolizumab konnte ein Trend Richtung weniger Nebenwirkungen gefunden werden und es wurde im Rahmen dieser Studie als das sicherste Präparat in Bezug auf schwere Nebenwirkungen angegeben als ,surface under the cumulative ranking curve" (SUCRA) mit 0,91 und Infektionskomplikationen (SUCRA: 0,75) gewertet [25].

Diese Daten unterstützt auch eine rezent publizierte Studie von Allamneni et al., in der die Wirksamkeit und das Sicherheitsprofil von Infliximab und Vedolizumab bei Colitis ulcerosa verglichen wurden. Von den eingeschlossenen 46 Patienten, die Infliximab erhielten, mussten 5 aufgrund von schweren Infektionen und anderen medikamentenassoziierten Komplikationen innerhalb der ersten 24 Therapiewochen aus der Studie ausgeschlossen werden. In der Vedolizumabgruppe mit 32 Patienten gab es keine vergleichbaren Komplikationen [26].

Wie zu erwarten zeigte Vedolizumab auch bei Morbus-Crohn-Patienten ein ausgezeichnetes Sicherheitsprofil. Colombel et al. publizierten rezent, dass bei 2830 Patienten aus 6 Studien unter Vedolizumab kein erhöhtes Infektionsrisiko bei Colitis ulcerosa und bei Morbus Crohn im Vergleich zu Placebo besteht [27].

Auch Mocko et al. fanden in einer Metaanalyse keine Häufung von schweren Nebenwirkungen bzw. von schweren Infektionen bei Vedolizumabtherapie im Vergleich zu Placebo bei Morbus Crohn [28].

In einem systematischen Review von Mocko et al. mit 10 eingeschlossenen Studien werden ebenfalls die Sicherheitsdaten der am Markt befindlichen Biologika für die Anwendung bei Morbus Crohn verglichen. Die Studie findet ein vergleichbares Sicherheitsprofil für alle Biologika in Hinsicht auf Nebenwirkungen, aber Vedolizumab kristallisiert sich 
als sicherstes Medikament in der Induktionsphase heraus [29].

Lukin et al. präsentierten im Rahmen der ECCO 2018 weitere Daten von 872 mit Biologika behandelten CED-Patienten, die dafür sprechen, dass Vedolizumab ein besseres Sicherheitsprofil als Anti-TNF-Inhibitoren aufweist. In der Vedolizumabkohorte traten schwere Infektionen seltener auf (6,9\% vs. $10,1 \%$; OR $0,67[0,41-1,07])$ und schwere therapieassoziierte Nebenwirkungen waren signifikant seltener $(7,1 \%$ vs. $13,1 \%$; OR $0,51[0,32-0,81])$ [30].

5. CED-Patienten mit einem Alter über 65 Lebensjahren mit Therapieversagen auf eine konventionelle Therapie sollten aufgrund des erhöhten Infektionsrisikos und der erhöhten Mortalität unter TNF-a-Inhibitoren primär mit Vedolizumab therapiert werden.

Wie bereits im vorigen Absatz erläutert liegen Hinweise vor, dass Vedolizumab hinsichtlich Nebenwirkungen und Infektionskomplikationen das derzeit sicherste CED-Biologikum am Markt $\mathrm{zu}$ sein scheint. Ältere Patienten sind aufgrund ihrer Konstitution und ihres Risikoprofils per se einem höheren Risiko für Infektionen ausgesetzt und sollten aus diesem Grund bei entsprechender Indikation mit dem sichersten Präparat (bei gleicher bis besserer Wirksamkeit) behandelt werden.

Ferner wurde für Colitis-ulcerosaoder Morbus-Crohn-Patienten über 65 Jahren mit laufender TNF- $\alpha$-Inhibitor-Therapie ein deutlich erhöhtes Infektions- und Todesrisiko verglichen zu Patienten über 65 Jahren und ohne TNF- $\alpha$-Therapie und zu Patienten unter 65 Jahren mit TNF- $\alpha$-Therapie gefunden. Schwere Infektionen traten bei $11 \%$ dieser Patienten auf, $10 \%$ verstarben in dieser italienischen Kohorte. Im Vergleich hierzu trat dies in den anderen beiden Gruppen deutlich seltener auf (Infektionen: $0,5 \%$ bei Alter $>65$ ohne TNF-Therapie; $2,6 \%$ bei Alter $<65$ mit TNF-Therapie; Tod: $2 \%$ bei Alter $>65$ ohne TNF-Therapie; $1 \%$ bei Alter $<65$ mit TNF-Therapie; [31]).

Für Vedolizumab hingegen liegen Daten bei Colitis ulcerosa und Morbus Crohn vor, die eine vergleichbare
Wirkung und ein vergleichbares Sicherheitsprofil im Alter über 55 Jahren zeigen [32].

Die gute Verträglichkeit von Vedolizumab im höheren Alter wird weiters mit kleineren Studien untermauert. So fanden Grace et al. in ihrer 79 Patienten umfassenden Kohorte von Colitis-ulcerosaund Morbus-Crohn-Patienten keine erhöhte Rate an schweren Nebenwirkungen in der Altersgruppe über 50 Jahren unter Vedolizumab-Therapie [33]. In einer weiteren Arbeit von Navaneethan et al. mit allerdings sehr kleiner Patientenzahl von nur 29 CED-Patienten ergab sich ebenfalls eine gute Verträglichkeit von Vedolizumab [34].

6. Vedolizumab sollte bei Kontraindikationen für eine TNF-a-InhibitorenTherapie als Erstlinienbiologikum eingesetzt werden. Laut den Fachinformationen der TNF- $\alpha$-Inhibitoren sind Patienten mit bekannter Unverträglichkeit auf diese Medikamentengruppe, mit florider Infektion und mit einer Herzinsuffizienz Grad III/IV von der Behandlung auszunehmen. Vorsicht gilt auch beim Einsatz bei Herzinsuffizienz Grad I/II, bei sämtlichen Infektionen inklusive Tuberkulose, bei multipler Sklerose oder Guillain-Barré-Syndrom sowie bei malignen und lymphoproliferativen Erkrankungen [35, 36].

Der Tumornekrosefaktor- $\alpha$ (TNF- $\alpha$ ) kann über Caspasen Zellapoptosen induzieren. Aufgrund dieser Tatsache könnte eine Inhibierung von TNF- $\alpha$ das Wachstum und die Metastasierung von Tumoren begünstigen. Im Gegenzug hierzu kann TNF- $\alpha$ über den NF- $\kappa$ B-Signalweg die Proliferation und das Überleben von neoplastischen Zellen begünstigen und eine Blockade dieses Signalwegs könnte antitumoröse Effekte haben. Allerdings sind weder wachstumsfördernde noch antitumoröse Effekte von TNF- $\alpha$-Inhibitoren bisher wirklich belegt. In einer retrospektiven Arbeit bei 108.579 CEDPatienten wurde aber für TNF- $\alpha$-Inhibitoren ein 1,9-fach erhöhtes Risiko für die Entwicklung von malignen Melanomen festgestellt [37]. In der Leitlinie „Inflammatory bowel disease and malignancies“ der ECCO wird aus diesem Grund von der Verwendung von TNF- $\alpha$-Inhibitoren bei Patienten mit malignen Melanomen in der Anamnese abgeraten [38].

Für Vedolizumab findet sich keine Kontraindikation für die Anwendung bei Herzinsuffizienz und bei demyelinisierenden Erkrankungen [1]. In Hinsicht auf floride Infektionen kann auch Vedolizumab nicht ohne Vorsicht eingesetzt werden. Aufgrund der darmselektiven Wirkung dürften allerdings auch bei dieser Indikation Vorteile für Vedolizumab bestehen (siehe oben). In der ECCOLeitlinie „Inflammatory bowel disease and malignancies“ [38] wird Vedolizumab und dessen Einsatz bei Malignomen noch nicht behandelt, die Zulassungsdaten zeigen allerdings kein erhöhtes Malignomrisiko unter der Therapie mit Vedolizumab [5, 18], Langzeitdaten und Erfahrungen bei Patienten mit Malignomen liegen jedoch nicht vor.

\section{Fazit für die Praxis}

Vedolizumab ist in der Induktionstherapie bei TNF-naiven Patienten mit nichtakuter mittelschwerer und schwerer Colitis ulcerosa gleich wirksam wie TNFa-Inhibitoren. Langfristig scheint Vedolizumab in Bezug auf die Effizienz sowie auf das Nebenwirkungsprofil Vorteile gegenüber einer TNF- $a$-InhibitorenTherapie zu besitzen. Auch bei TNFnaiven Patienten mit Morbus Crohn ist Vedolizumab in der Erhaltungstherapie eine ähnlich wirksame Alternative zu anderen Biologika. Die Möglichkeit einer Erstlinientherapie soll daher gegeben sein. Dies gilt insbesondere für Subgruppen wie ältere Patienten oder Patienten mit erhöhtem Infektionsrisiko.

\section{Korrespondenzadresse

Dr. Andreas Blesl
Klinische Abteilung für
Gastroenterologie und
Hepatologie, Medizinische
Universität Graz
Auenbruggerplatz 15,
8036 Graz, Österreich
andreas.blesl@medunigraz.at

Förderung. Das Zustandekommen dieser Publikation erfolgte auf Initiative der AG CED und wurde unterstützt von Takeda Pharma GmbH. 
Funding. Open access funding provided by Medical University of Graz.

\section{Einhaltung ethischer Richtlinien}

Interessenkonflikt. A. Blesl erhielt Honorare für Beratertätigkeit und Vorträge von den Firmen Abbvie, Janssen, Takeda, Genericon und Vifor. C. Högenauer erhielt Honorare für Beratertätigkeit und Vorträge von den Firmen Abbvie, Astro-Pharma, Falk, Ferring, Janssen, MSD, Takeda, Pfizer und Vifor. R. Koch erhielt Honorare für Beratertätigkeit und Vorträge von den Firmen Abbvie, Astro-Pharma, Hospira, Janssen, Merck, MSD und Takeda. C. Dejaco erhielt Honorare fü Beratertätigkeit und Vorträge von den Firmen Abbvie, Astro-Pharma, Janssen, MSD, Takeda, Pfizer und Vifor. $\mathrm{H}$. Vogelsang erhielt Honorare für Beratertätigkeit und Vorträge von den Firmen Abbvie, Astro-Pharma, Falk, Ferring, Janssen, MSD, Shire, Takeda, Pfizer und Vifor. J.Stimakovits erhielt Honorare für Beratertätigkeit und Vorträge von den Firmen Astro-Pharma, Janssen und Takeda. H. Fuchssteiner erhielt Honorare für Beratertätigkeit und Vorträge von den Firmen Abbvie, Janssen und Takeda; T. Haas: Abbvie, Astro-Pharma, Janssen, MSD und Takeda. W. Petritsch erhielt Honorare für Beratertätigkeit und Vorträge von den Firmen Abbvie, Astro-Pharma, Falk, Ferring, Janssen, MSD, Takeda und Vifor.

Dieser Beitrag beinhaltet keine von den Autoren durchgeführten Studien an Menschen oder Tieren.

Open Access Dieser Artikel wird unter der Creative Commons Namensnennung 4.0 International Lizenz (http://creativecommons.org/licenses/by/4.0/deed. de) veröffentlicht, welche die Nutzung, Vervielfältigung, Bearbeitung, Verbreitung und Wiedergabe in jeglichem Medium und Format erlaubt, sofern Sie den/die ursprünglichen Autor(en) und die Quelle ordnungsgemäßnennen, einen Link zur Creative Commons Lizenz beifügen und angeben, ob Änderungen vorgenommen wurden.

\section{Literatur}

1. EMA Entyvio, product information. http:// www.ema.europa.eu/ema/index.jsp?curl=pages/ medicines/human/medicines/002782/human med_001751.jsp\&mid=WC0b01ac058001d124 Accessed.Zugegriffen:27.Jan. 2018

2. Harbord M, Eliakim R, Bettenworth D, Karmiris K, Katsanos K, Kopylov U, KucharzikT, Molnár T, Raine T, Sebastian S, de Sousa HT, Dignass A, Carbonnel F, European Crohn's and Colitis Organisation [ECCO] (2017) Third European evidence-based consensus on diagnosis and management of ulcerative colitis. Part 2: current management. J Crohns Colitis 11(7):769-784. https://doi.org/10.1093/ecco-jcc/ jjx009

3. Gomollón F, Dignass A, Annese V, Tilg H, Van Assche G, Lindsay JO, Peyrin-Biroulet L, Cullen GJ, Daperno M, Kucharzik T, Rieder F, Almer S, Armuzzi A, Harbord M, Langhorst J, Sans M, Chowers $Y$, Fiorino $G$, Juillerat $P$, Mantzaris GJ, Rizzello $F$, Vavricka S, Gionchetti P, ECCO (2017) 3rd European evidence-based consensus on the diagnosis and management of Crohn's disease 2016: part 1: diagnosis and medical management. J Crohns Colitis 11(1):3-25. https://doi.org/10.1093/eccojcc/jjw168
4. Novacek G, Dejaco C, Gröchenig HP, Högenauer C, Knoflach P, Miehsler W, Moschen A, Petritsch W, Vogelsang H, Tilg H (2016) Vedolizumab in der Behandlung der Colitis ulcerosa und des Morbus Crohn - ein Konsensus der Arbeitsgruppe "Chronisch Entzündliche Darmerkrankungen" der Österreichischen Gesellschaft für Gastroenterologie und Hepatologie. Z Gastroenterol 54(5):456-467. https://doi.org/10.1055/s-0042103694

5. Feagan BG, Rutgeerts $P$, Sands BE, Hanauer $S$, Colombel JF, Sandborn WJ, Van Assche G, Axler J, Kim HJ, Danese S, Fox I, Milch C, Sankoh S, Wyant T, Xu J, Parikh A, GEMINI 1 Study Group (2013) Vedolizumab as induction and maintenance therapy for ulcerative colitis. $\mathrm{N}$ Engl J Med 369(8):699-710. https://doi.org/10.1056/ NEJMoa1215734

6. Rutgeerts P, Sandborn WJ, Feagan BG, Reinisch W, Olson A, Johanns J, Travers S, Rachmilewitz D, Hanauer SB, Lichtenstein GR, de Villiers WJ, Present D, Sands BE, Colombel JF (2005) Infliximab for induction and maintenance therapy for ulcerative colitis. N Engl J Med 353(23):2462-2476

7. Reinisch W, Sandborn WJ, Hommes DW, D'Haens G, Hanauer S, Schreiber S, Panaccione R, Fedorak RN, Tighe MB, Huang B, Kampman W, Lazar A, Thakkar R (2011) Adalimumab for induction of clinical remission in moderately to severely active ulcerative colitis: results of a randomised controlled trial. Gut 60(6):780-787. https://doi. org/10.1136/gut.2010.221127

8. Sandborn WJ, van Assche G, Reinisch W, Colombel JF, D'Haens G, Wolf DC, Kron M, Tighe MB, Lazar A, Thakkar RB (2012) Adalimumab induces and maintains clinical remission in patients with moderate-to-severe ulcerative colitis. Gastroenterology 142(2):257-265.e1-3. https://doi.org/10.1053/j. gastro.2011.10.032

9. Sandborn WJ, Feagan BG, Marano C, Zhang H, Strauss R, Johanns J, Adedokun OJ, Guzzo C, Colombel JF, Reinisch W, Gibson PR, Collins J, Järnerot G, Hibi T, Rutgeerts P, PURSUIT-SC Study Group (2014) Subcutaneous golimumab induces clinical response and remission in patients with moderate-to-severe ulcerative colitis. Baillieres Clin Gastroenterol 146(1):85-95. https://doi.org/ 10.1053/j.gastro.2013.05.048 (quize 14-5)

10. Feagan BG, Rubin DT, Danese $S$, Vermeire $S$, Abhyankar B, Sankoh S, James A, Smyth M (2017) Efficacy of Vedolizumab induction and maintenance therapy in patients with ulcerative colitis, regardless of prior exposure to tumor necrosis factor antagonists. Clin Gastroenterol Hepatol 15(2):229-239.e5. https://doi.org/10. 1016/j.cgh.2016.08.044

11. Cholapranee A, Hazlewood GS, Kaplan GG, PeyrinBiroulet L, Ananthakrishnan AN (2017) Systematic review with meta-analysis: comparative efficacy of biologics for induction and maintenance of mucosal healing in Crohn's disease and ulcerative colitis controlled trials. Aliment Pharmacol Ther 45(10):1291-1302. https://doi.org/10.1111/apt. 14030

12. Danese S, Fiorino G, Peyrin-Biroulet L, Lucenteforte E, Virgili G, Moja L, Bonovas S (2014) Biological agents for moderately to severely active ulcerative colitis: a systematic review and network metaanalysis. Ann Intern Med 160(10):704-711

13. Sandborn WJ, Colombel JF, D'Haens G, Van Assche G, Wolf D, Kron M, Lazar A, Robinson AM, Yang M, Chao JD, Thakkar R (2013) One-year maintenance outcomes among patients with moderately-toseverely active ulcerative colitis who responded to induction therapy with adalimumab: subgroup analyses from ULTRA 2. Aliment Pharmacol Ther 37(2):204-213. https://doi.org/10.1111/apt. 12145

14. Sandborn WJ, Feagan BG, Marano $C$, Zhang $H_{\text {, }}$ Strauss R, Johanns J, Adedokun OJ, Guzzo C, Colombel JF, Reinisch W, Gibson PR, Collins J, Järnerot G, Rutgeerts P, PURSUIT-Maintenance Study Group (2014) Subcutaneous golimumab maintains clinical response in patients with moderate-to-severe ulcerative colitis. Baillieres Clin Gastroenterol 146(1):96-109.e1. https://doi. org/10.1053/j.gastro.2013.06.010

15. Vickers AD, Ainsworth $C$, Mody R, Bergman A Ling CS, Medjedovic J, Smyth M (2016) Systematic review with network meta-analysis: comparative efficacy of biologics in the treatment of moderately to severely active ulcerative colitis. PLoS ONE 11(10):e165435. https://doi.org/10.1371/journal. pone. 0165435

16. Faleck $D$, Shashi $P$, Meserve J, Rahal M, Kadire $S$, Tran G, Weiss A, Winters A, Chablaney S, Aniwan S, Koliani-Pace JL, Kochhar G, Boland B, Singh S, Hirten R, ShmidtE, Lasch K, Luo M, Bohm M, Sagi SV, Fischer M, Hudesman D, Chang S, Lukin D, Sultan K, Swaminath A, Gupta N, SiegelCA, Shen B, Sandborn WJ, Sands BE, Colombel J-F, Kane S, Loftus EV, Dulai PS (2018) OP026 comparative effectiveness of vedolizumab and TNF-antagonist therapy in ulcerative colitis: a multicentre consortium propensity score-matched analysis. J Crohns Colitis. https://doi.org/10.1093/ecco-jcc/jjx180. 025

17. Scott FI, Shah Y, Lasch K, Luo M, Lewis JD (2018) Assessing the optimal position for Vedolizumab in the treatment of ulcerative colitis: a simulation model. Inflamm Bowel Dis 24(2):286-295

18. Sands BE, Sandborn WJ, Van Assche G, Lukas M, Xu J, James A, Abhyankar B, Lasch KS (2017) Vedolizumab as induction and maintenance therapy for Crohn's disease in patients naïve to or who have failed tumor necrosis factor antagonist therapy. Inflamm Bowel Dis 23(1):97-106

19. Danese S, Feagan B, Sandborn W, Colombel J-F, Vermeire S, Jones S, Brennan K, Bornstein J (2018) A phase $3 \mathrm{~b}$ open-label multicentre study (VERSIFY) of the efficacy of vedolizumab on endoscopic healing in moderately to severely active Crohn's Disease (CD). J Crohns Colitis. https://doi.org/10 1093/ecco-jcc/jjx180.022

20. Singh S, Garg SK, Pardi DS, Wang Z, Murad MH, Loftus EV Jr. (2014) Comparative efficacy of biologic therapy in biologic-naïve patients with Crohn disease: a systematic review and network meta-analysis. Mayo Clin Proc 89(12):1621-1635. https://doi.org/10.1016/j.mayocp.2014.08.019

21. Hazlewood GS, Rezaie A, Borman M, Panaccione R, Ghosh S, Seow CH, Kuenzig E, Tomlinson G, Siegel CA, Melmed GY, Kaplan GG (2015) Comparative effectiveness of immunosuppressants and biologics for inducing and maintaining remission in Crohn's disease: a network meta-analysis. Gastroenterology 148(2):344-354.e5. https://doi.org/10.1053/j. gastro.2014.10.011 (quize14-5)

22. Bohm M, Sagi SV, Fischer M, Kadire S, Tran G, Rahal M, Aniwan S, Meserve J, Weiss A, Kochhar G, Shashi P, Faleck D, Winters A, Chablaney S, KolianiPace JL, Boland B, Singh S, Hirten R, Shmidt E, Lasch K, Luo M, Hudesman D, Chang S, Lukin D, Sultan K, Swaminath A, Gupta N, Siegel CA, Kane $S$, Loftus EV, Sands BE, Sandborn WJ, Colombe J-F, Shen B, Dulai PS (2018) OP025 Comparative effectiveness of vedolizumab and tumour necrosis factor-antagonist therapy in Crohn's disease: 
a multicentre consortium propensity scorematched analysis. J Crohns Colitis. https://doi.org/ 10.1093/ecco-jcc/jjx180.024

23. Kawalec $P$, Moćko P (2018) An indirect comparison of ustekinumab and vedolizumab in the therapy of TNF-failure Crohn's disease patients. J Comp EffRes 7(2):101-111. https://doi.org/10.2217/cer-20170041

24. FaleckD, Winters A, Chablaney S, Shashi P, Meserve J, Weiss A, Aniwan S, Koliani-Pace JL, Kochhar G, Boland B, Singh S, Hirten R, Shmidt E, Lasch K, Luo M, Bohm M, Sagi SV, Fischer M, Hudesman D, Chang S, Lukin D, Sultan K, Swaminath A, Gupta N, Siegel CA, Shen B, Sandborn WJ, Kane S, Loftus EV, Sands BE, Colombel J-F, Dulai PS, Ungaro R (2018) DOP051 Shorter disease duration is associated with higher response rates to vedolizumab in Crohn's disease but not ulcerative colitis: a multicentre consortium analysis. J Crohns Colitis. https://doi.org/10.1093/ecco-jcc/jjx180.088

25. Singh S, Fumery M, Sandborn WJ, Murad MH (2018) Systematic review with network metaanalysis: first- and second-line pharmacotherapy for moderate-severe ulcerative colitis. Aliment Pharmacol Ther 47(2):162-175. https://doi.org/ 10.1111/apt.14422

26. Allamneni C, Venkata K, Yun H, Xie F, DeLoach $L$, Malik TA (2018) Comparative effectiveness of Vedolizumab vs. Infliximab induction therapy in ulcerative colitis: experience of a real-world cohort at a tertiary inflammatory bowel disease center. Gastroenterology Res 11(1):41-45. https://doi. org/10.14740/gr934w

27. Colombel JF, Sands BE, Rutgeerts P, Sandborn W, Danese S, D'Haens G, Panaccione R, Loftus EV Jr, Sankoh S, Fox I, Parikh A, Milch C, Abhyankar B, Feagan BG (2017) The safety of vedolizumab for ulcerative colitis and Crohn's disease. Gut 66(5):839-851. https://doi.org/10.1136/gutjnl2015-311079

28. Moćko P, Kawalec P, Smela-Lipińska B, Pilc A (2016) Effectiveness and safety of vedolizumab for treatment of Crohn's disease: a systematic review and meta-analysis. Arch Med Sci 12(5):1088-1096

29. Moćko P, Kawalec P, Pilc A (2016) Safety profile of biologic drugs in the therapy of Crohn disease: a systematic review and network meta-analysis. Pharmacol Rep 68(6):1237-1243. https://doi.org/ 10.1016/j.pharep.2016.07.013

30. Lukin D, Weiss A, Aniwan S, Kadire S, Tran G, Raha M, Faleck D, Winters A, Chablaney S, Meserve J, Kochhar G, Shashi P, Koliani-Pace JL, Bohm M, Sagi SV, Fischer $M$, Boland $B$, Singh $S$, Hirten R, Shmidt E, Hudesman D, Chang S, Sultan K, Swaminath A, Gupta N, Kane S, Loftus EV, Shen B, Sands BE, Sandborn WJ, Colombel J-F, Siegel CA, Dulai PS (2018) DOP009 comparative safety profile of vedolizumab and tumour necrosis factor-antagonist therapy for inflammatory bowel disease: a multicentre consortium propensity score-matched analysis. J Crohns Colitis. https:// doi.org/10.1093/ecco-jcc/jjx180.046

31. Cottone M, Kohn A, Daperno M, Armuzzi A, Guidi $L$, D'Inca R, Bossa F, Angelucci $E$, Biancone $L$, Gionchetti P, Ardizzone S, Papi C, Fries W, Danese S, Riegler G, Cappello M, Castiglione F, Annese V, Orlando A (2011) Advanced age is an independent risk factor for severe infections and mortality in patients given anti-tumor necrosis factor therapy forinflammatory bowel disease. Clin Gastroenterol Hepatol 9(1):30-35.https://doi.org/10.1016/j.cgh 2010.09.026

32. Yajnik V, Khan N, Dubinsky M, Axler J, James A, Abhyankar B, Lasch K (2017) Efficacy and safety of Vedolizumab in ulcerative colitis and Crohn's disease patients stratified by age. Adv Ther 34(2):542-559. https://doi.org/10.1007/s12325016-0467-6

33. Grace R et al (2015) Infectious complications in IBD patients on immunomodulators, corticosteroids, and vedolizumab: is older age a predictor of higher complication rates or worsened response? Am J Gastroenterol 110(Suppl 1):S823 (Abstract 1940)

34. Navaneethan U, Edminister T, ZhuX, Kommaraju K, Glover S (2017) Vedolizumab is safe and effective in elderly patients with inflammatory bowel disease. Inflamm Bowel Dis 23(4):E17

35. Fachinformation Humira. http://www. ema.europa.eu/ema/index.jsp?curl=pages/ medicines/human/medicines/000481/human med_000822.jsp\&mid=WC0b01ac058001d124. Zugegriffen:6. Febr. 2018

36. Fachinformation Remicade. http://www. ema.europa.eu/ema/index.jsp?curl=pages/ medicines/human/medicines/000240/human med_001023.jsp\&mid=WCOb01ac058001d124. Zugegriffen:6. Febr. 2018

37. Long MD, Martin CF, Pipkin CA, Herfarth $\mathrm{HH}$, Sandler RS, Kappelman MD (2012) Risk of melanoma and nonmelanoma skin cancer among patients with inflammatory bowel disease. Gastroenterology 143(2):390-399.e1. https://doi. org/10.1053/j.gastro.2012.05.004

38. Annese V, Beaugerie L, Egan L, Biancone L, Bolling C, Brandts C, Dierickx D, Dummer R, Fiorino G Gornet JM, Higgins P, Katsanos KH, Nissen L, Pellino G, Rogler G, Scaldaferri F, Szymanska E, Eliakim R, ECCO (2015) European evidence-based consensus: inflammatory bowel disease and malignancies. JCrohns Colitis 9(11):945-965 